\title{
Retrosternal and pleural "bridging" fibrosis mimicking malignancy in a patient exposed to asbestos
}

\author{
M-P. Debray*, S. Marchand-Adam\#", R. Soufan`, J. Ostinelli', P-Y. Brillet ${ }^{\S}$, \\ E. Schouman-Claeys* and B. Crestani ${ }^{+}$
}

ABSTRACT: Mediastinal fibrosis is a rare, benign disorder characterised by proliferation of fibrous tissue within the mediastinum. It can present as diffuse mediastinal infiltration or, more frequently, as a localised soft tissue mass, usually in the right paratracheal or subcarinal areas. 18-fluorodeoxyglucose (18-FDG) positron emission tomography (PET) imaging has only been reported in a few case reports with variable aspects.

Herein, we present an unusual pattern of mediastinal fibrosis, presenting as a thick retrosternal mass contiguous with pleural thickening. 18-FDG PET showed intensely increased uptake in the mass. Both the location of fibrosis and PET imaging were confusing with malignancy. Histopathological proof of benign fibrosis was obtained by surgery. The patient had been exposed to asbestos and the possible relationship of this particular pattern of fibrosis with asbestos exposure will be discussed.

KEYWORDS: Asbestos exposure, computed tomography, mediastinal fibrosis, positron emission tomography

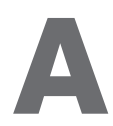
64-yr-old nonsmoking male was evaluated for recent retrosternal pain in the context of past exposure to asbestos, having worked as a house and spray painter for 30 yrs. He had a history of regressive left lymphocytic pleural effusion with mediastinal adenomegaly, which was diagnosed in 1994. In 2002, he received anti-tuberculous treatment for a right pleural effusion without a definitive proof of tuberculosis. The computed tomography (CT) scan at that time showed a moderate right pleural effusion, without pleural thickening (fig. 1). A mild right pleural opacity was subsequently seen on plain chest radiographs. At presentation he was complaining of recent retrosternal pain, thus, a CT scan was performed in May 2007. The CT scan revealed a $20-\mathrm{mm}$ thick noncalcified homogeneous elongated retrosternal mass, which showed very mild enhancement after intravenous injection of iodinated contrast media. The mass extended from one pleural membrane to the other and was contiguous with a regular noncalcified thickening of the right costal pleura (fig. 2). Moderately hypertrophied mediastinal adenopathies were present. There were no signs of sternal erosion or sclerosis, and no signs of parietal muscles invasion. An abdominal CT scan was normal. Physical examination did not reveal any extra thoracic signs. Standard blood tests were within the normal range. Antinuclear antibodies were positive to $1 / 160$ with no specificity identified, and rheumatoid factor was negative. Lung function tests revealed a severe restrictive impairment (total lung capacity $49 \%$ of predicted). Positron emission tomography (PET) showed markedly homogeneously increased uptake of 18-fluorodeoxyglucose (18-FDG) in the retrosternal mass (with the maximum standard uptake value being 5) and a mildly increased uptake in the pleural thickening (fig. 3). There was no extrathoracic abnormal uptake. A malignant tumour was suspected and a large surgical resection of the mass was performed in July 2007. The results showed a firm whitish homogeneous mass infiltrating both adipose and muscular tissue at the periphery. Histopathological analysis revealed extensive fibrous tissue infiltrated by inflammatory cells in varying proportion with some lymphoid follicles at the periphery consistent with the
AFFILIATIONS

${ }^{*}$ Depts of Radiology,

'Pathology,

+Pneumology, Groupe Hospitalier

Bichat Claude Bernard, Assistance Publique-Hôpitaux de Paris,

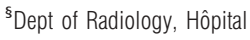

Avicenne, Assistance Publique-

Hôpitaux de Paris, Paris, and

\#Dept of Pneumology, Hôpital

Bretonneau, Tours, France.

CORRESPONDENCE

M-P Debray

Dept of Radiology

Groupe Hospitalier Bichat Claude Bernard

46 rue Henri Huchard

75877 Paris

Cedex 18

France

E-mail: marie-pierre.debray@

bch.aphp.fr

Received:

Jan 052010

Accepted after revision:

Feb 082010

PROVENANCE

Submitted article, peer reviewed.

European Respiratory Review

Print ISSN 0905-9180

Online ISSN 1600-0617 


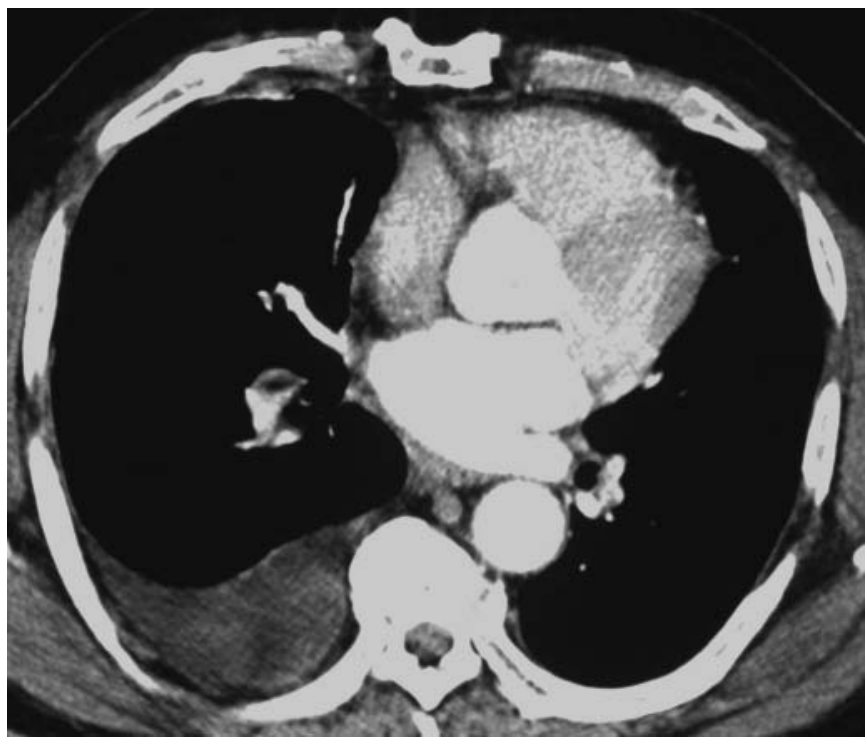

FIGURE 1. Contrast-enhanced computed tomography of the chest performed in 2002 showing a moderate right pleural effusion without clear pleural thickening or mediastinal mass.

diagnosis of fibrosing mediastinitis (fig. 4). No tumoural cells, necrosis or infectious agents were present. No recurrence of the lesion was detected on a thoracic CT scan performed 4 months later.

\section{DISCUSSION}

In this patient, fibrosing mediastinitis appeared as a large elongated mass localised in the retrosternal area. As a focal lesion, it belongs to the group of focal forms of this entity [1].
These most commonly affect the middle mediastinal compartment and are frequently calcified. Compression of mediastinal structures, such as the central airways, central veins, pulmonary arteries or oesophagus, frequently reveals the disease. Localised involvement of the retrosternal area has been reported recently in two patients with retrosternal "bridging" fibrosis, extending from one pleural membrane to the other and contiguous with pleural thickening [2], a similar pattern to that seen in our patient. However, contrary to our patient, these cases showed associated retroperitonal fibrosis and no or slightly increased uptake of 18-FDG on PET imaging. COTTIN et al. [2] raised the possible role of asbestos exposure in the development of the fibrosis. The patient we describe herein was also exposed to asbestos which, retrospectively, probably explained the recurrent pleural effusions that he experienced in the past 13 yrs. As he received anti-tuberculous therapy in 2002 , no bacteriological proof of mycobacterial infection could be established at that time. Tuberculosis, an established cause of mediastinal fibrosis, thus seems an unlikely cause in our patient and the possible role of asbestos exposure in the development of such particular retrosternal and pleural "bridging" fibrosis can be considered.

The anatomic continuity of the mass with pleural thickening raises the question of the pathophysiology of this particular form of mediastinal fibrosis. As pleural thickening preceded the discovery of the mass, it can not be excluded that the latter is a mediastinal extension of pleural fibrosis.

The first diagnosis that should be excluded in the presence of a soft tissue retrosternal mass is lymphoma. Other possible aetiologies include sarcoma, thymoma and germ cell tumours. Owing to the continuity of the mass with pleural thickening and in the setting of exposure to asbestos, mesothelioma
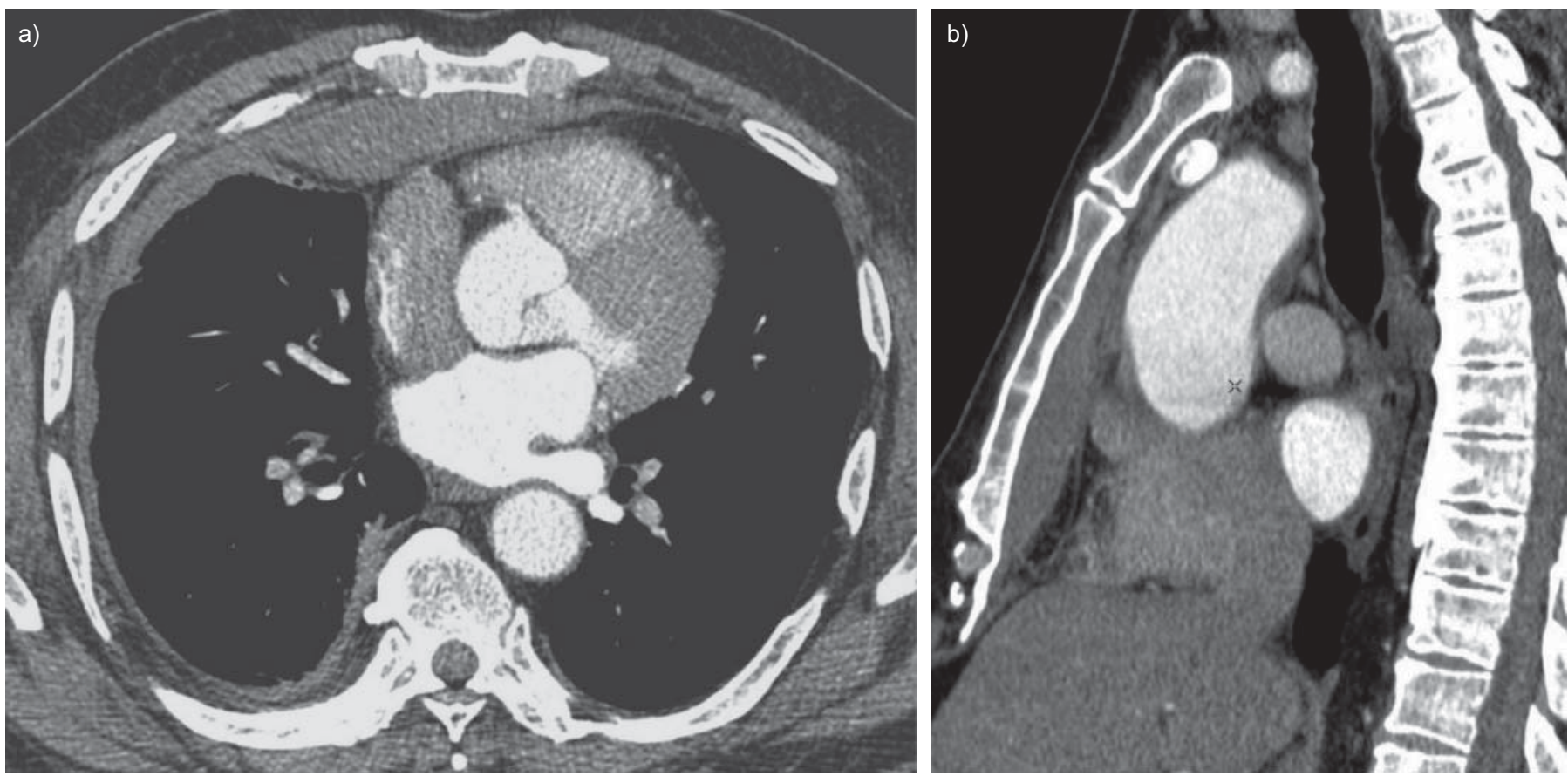

FIGURE 2. Contrast-enhanced computed tomography of the chest in the a) axial and b) sagittal planes. Computed tomography was performed in May 2007 when the patient complained of retrosternal pain. The images demonstrate an elongated retrosternal mass with regular borders merging with a thick right pleural thickening 


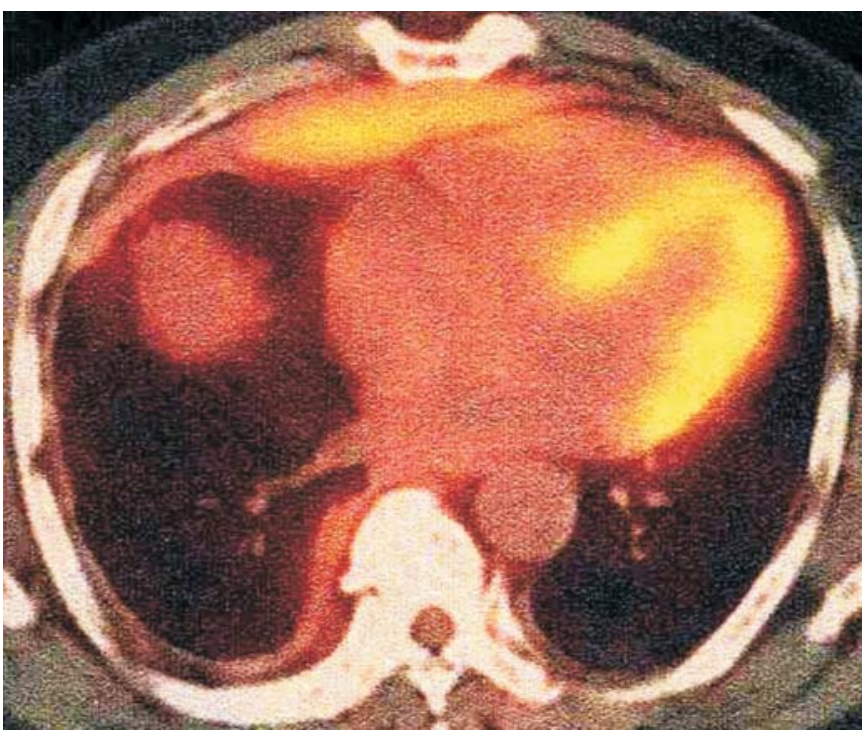

FIGURE 3. Positron emission tomography scan in the axial plane performed in June 2007 showing intense and homogeneous increased uptake of 18-fluorodeoxyglucose within the mass (with a standard uptake value of 5). Note the physiological increased uptake of the myocardium.

should also be considered in our patient. Because fibrosis may be associated with most of these tumours, small biopsy samples obtained with a percutaneous needle should be interpreted with caution when they show fibrosis. Large open biopsies are then necessary to make the final diagnosis.

Increased 18-FDG uptake in the mass was particularly confusing in our patient as many tumours of the anterior mediastinum, especially lymphoma, are associated with an increased uptake of 18-FDG. 18-FDG PET imaging for fibrosing mediastinitis has only been reported in case reports with increased uptake in some cases [3] and no uptake in others [4]. Such differences could be attributed to various histopathological patterns of fibrosis. Indeed, FLIEDER et al. [5] described a histopathological spectrum of mediastinal fibrosis which they classified in three stages; from oedematous fibromyxoid tissue in stage I to dense paucicellular collagen bundles in stage III. Our case presents with findings in the middle of this classification, between stage I and II. Despite this, the classification has not been correlated to 18-FDG PET imaging. Accumulation of FDG in young granulation tissue is well documented and we think the increased uptake seen in our

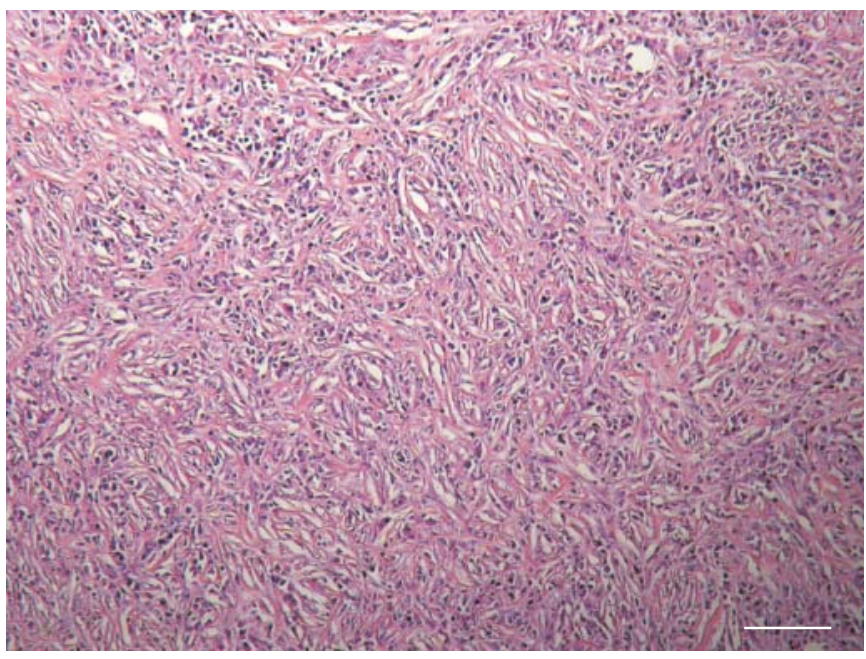

FIGURE 4. Photomicrograph of the mass removed from surgery showing numerous inflammatory cells interspersed with collagen bundles consistent with mediastinal fibrosis. Haematoxylin and eosin stain. Scale bar $=200 \mu \mathrm{m}$.

patient could be attributed to numerous inflammatory cells and small blood vessels homogeneously distributed in the mass.

In conclusion, mediastinal fibrosis can develop as a localised retrosternal mass mimicking malignancy and this pattern may be related to asbestos exposure.

\section{STATEMENT OF INTEREST}

J. Ostinelli is a full time employee of AstraZeneca (Rueil-Malmasion, France) and has stocks in excess of $€ 5,000$ in AstraZeneca.

\section{REFERENCES}

1 Rossi SE, McAdams HP, Rosado-de-Christenson ML, et al. Fibrosing mediastinitis. Radiographics 2001; 21: 737-757.

2 Cottin V, Brillet PY, Combarnous F, et al. Syndrome of pleural and retrosternal "bridging" fibrosis and retroperitoneal fibrosis in patients with asbestos exposure. Thorax 2008; 63: 177-179.

3 Nakajo M, Jinnouchi S, Tanabe H, et al. 18F-fluorodeoxyglucose positron emission tomography features of idiopathic retroperitoneal fibrosis. J Comput Assist Tomogr 2007; 31: 539-543.

4 Chong S, Kim TS, Kim BT, et al. Fibrosing mediastinitis mimicking malignancy at CT: negative FDG uptake in integrated FDG PET/CT imaging. Eur Radiol 2007; 17: 1644-1646.

5 Flieder DB, Suster S, Moran CA. Idiopathic fibroinflammatory lesions of the mediastinum: a study of 30 cases with emphasis on morphologic heterogeneity. Mod Pathol 1999; 12: 257-264. 